\title{
Article \\ Chemotaxonomic Classification of Peucedanum japonicum and Its Chemical Correlation with Peucedanum praeruptorum, Angelica decursiva, and Saposhnikovia divaricata by Liquid Chromatography Combined with Chemometrics
}

\author{
Jung-Hoon Kim ${ }^{1,+} \mathbb{D}$, Eui-Jeong Doh ${ }^{2,+}$ and Guemsan Lee $2,3, * \mathbb{D}$ \\ 1 Division of Pharmacology, School of Korean Medicine, Pusan National University, Yangsan 50612, Korea; \\ kmsct@pusan.ac.kr \\ 2 Research Center of Traditional Korean Medicine, Wonkwang University, Iksan 54538, Korea; \\ bluemoon-lion@hanmail.net \\ 3 Department of Herbology, College of Korean Medicine, Wonkwang University, Iksan 54538, Korea \\ * Correspondence: rasfin@wku.ac.kr; Tel.: +82-63-850-6985 \\ + These authors contributed equally to this work.
}

check for updates

Citation: Kim, J.-H.; Doh, E.-J.; Lee, G. Chemotaxonomic Classification of Peucedanum japonicum and Its

Chemical Correlation with

Peucedanum praeruptorum, Angelica decursiva, and Saposhnikovia divaricata by Liquid Chromatography Combined with Chemometrics. Molecules 2022, 27, 1675. https:// doi.org/10.3390/molecules27051675

Academic Editors: Maria Carla Marcotullio, Francesco Cacciola and José Sousa Câmara

Received: 29 December 2021 Accepted: 22 February 2022 Published: 3 March 2022

Publisher's Note: MDPI stays neutral with regard to jurisdictional claims in published maps and institutional affiliations.

Copyright: (C) 2022 by the authors. Licensee MDPI, Basel, Switzerland. This article is an open access article distributed under the terms and conditions of the Creative Commons Attribution (CC BY) license (https:// creativecommons.org/licenses/by/ $4.0 /)$.

\begin{abstract}
The roots of Peucedanum japonicum (Apiaceae) have been used as an alternative to the roots of Saposhnikovia divaricata (Apiaceae) to treat common cold-related symptoms in Korea. However, a variety of Peucedanum species, including the roots of P. praeruptorum or Angelica decursiva (=P. decursioum), have been used to treat phlegm-heat-induced symptoms in China. Hence, as there are differences in the medicinal application of P. japonicum roots between Korea and China, chemotaxonomic classification of $P$. japonicum was evaluated. Sixty samples derived from $P$. japonicum, P. praeruptorum, A. decursiva, and $S$. divaricata were phylogenetically identified using DNA barcoding tools, and chemotaxonomic correlations among the samples were evaluated using chromatographic profiling with chemometric analyses. P. japonicum samples were phylogenetically grouped into the same cluster as P. praeruptorum samples, followed by $S$. divaricata samples at the next cluster level, whereas $A$. decursiva samples were widely separated from the other species. Moreover, P. japonicum samples showed higher chemical correlations with P. praeruptorum samples or A. decursiva samples, but lower or negative chemical correlations with $S$. divaricata samples. These results demonstrate that P. japonicum is more genetically and chemically relevant to P. praeruptorum or A. decursiva and, accordingly, the medicinal application of P. japonicum might be closer to the therapeutic category of these two species than that of $S$. divaricata.
\end{abstract}

Keywords: Peucedanum japonicum; Peucedanum praeruptorum; Angelica decursiva; Saposhnikovia divaricata; genetic authentication; chemotaxonomic correlation

\section{Introduction}

Bang-Pung (Saposhnikoviae Radix), a traditional herbal medicine, has been used to treat common cold-induced disorders and relieve pain, and it originates from the roots of Saposhnikovia divaricata (Turcz.) Schischk. (Apiaceae) in Korean and Chinese pharmacopieas [1,2]. The roots of Peucedanum japonicum Thunb. (Apiaceae) are exclusively registered as "Sik-Bang-Pung" (Peucedani Japonici Radix; "Sik" means cultivated) in the Korean herbal pharmacopeia [3]. As the roots of P. japonicum are recognized as an alternative to Bang-Pung (the roots of $S$. divaricata), it is interesting that two different herbs belonging to two separate genera are used for the same medicinal purpose. Another notable issue is that the plant $P$. japonicum and its roots are botanically and medicinally named "Bin-Hae-Jeon-Ho" (bin hai qian hu; "Bin-Hae" means the plant lives near coastal areas), a type of "Jeon-Ho" in the Chinese literature [4,5]. Jeon-Ho, a medicinal name of Peucedani Radix, has been used to treat phlegm- and heat-induced respiratory disorders 
and it originates from the roots of P. praeruptorum Dunn (Baek-hwa-Jeon-Ho; "Baek-Hwa" means a white flower) or Angelica decursiva (Miq.) Franch. \& Sav. (=P. decursivum (Miq.) Maxim.) (Ja-Hwa-Jeon-Ho; "Ja-Hwa" refer to a purple flower) [2,3]. Hence, whether the roots of $P$. japonicum can be used for different therapeutic purposes in Korea and China is controversial. This is because the roots of $P$. japonicum are used for treatment of common cold-related symptoms in Korea, while different Peucedanum species, including P. japonicum, are currently used to treat phlegm- and heat-induced respiratory symptoms in China [5]. Therefore, it is necessary to classify the roots of $P$. japonicum for medicinal purposes. Moreover, an investigation of the chemical relationship between $P$. japonicum and $S$. divaricata, $P$. praeruptorum, and $A$. decursiva can be crucial to estimate the therapeutic efficacy-based medicinal categorization of P. japonicum.

There are several types of adulterants still distributed in the market for various reasons, contributing to confusion owing to the differences in origin species depending on the country and morphological similarity, and misunderstandings also originate from common names of plants and their medicinal names. Various research methods have been applied to prevent this confusion. DNA-based genetic analysis is one such approach. This molecular biological research method targeting genomic DNA is not affected by the environment, and the reliability is high because of the clarity and reproducibility of the analyzed results [6].

In previous studies using DNA-based approaches for S. divaricata, P. japonicum, and Glehnia littoralis, restriction fragment length polymorphism analysis was performed using the international transcribed spacer (ITS) primer for genomic DNA [7], and random amplified polymorphic DNA (RAPD) analysis-based sequence characterized amplified region markers were developed [8]. DNA barcode sequence (ITS and chloroplast DNA region) analysis has also been reported [9]. For Peucedani Radix, several DNA-based approaches, RAPD analysis [10], and ITS nucleotide analysis [11,12] have already been performed. Recently, the chloroplast genome has been used to understand the identities, phylogeny, genetic populations, and evolution of plants using next-generation sequencing $[13,14]$. These results demonstrated that DNA-based gene differentiation is an efficient and accurate method for applications. Therefore, DNA barcode analysis, comprising ITS combined with four chloroplast DNA regions, was performed for the identification of samples used in this study.

Chemotaxonomic classification of the four aforementioned species using the chromatographic profiling method with chemometric analysis is a logical approach to investigate the chemical relationship between $P$. japonicum and the remaining three species. The chemical classification of $S$. divaricata and P. japonicum was performed by metabolic profiling analysis using ultra-performance liquid chromatography/quadrupole time-of-flight mass spectrometry and multivariate analysis [15]. S. divaricata root and its substitute, P. ledebourielloides root, have previously been distinguished using liquid chromatography/mass spectrometrybased metabolomics [16]. A high-performance liquid chromatography/photodiode array detector was used for the quantitative analysis of the marker compounds and the chemical differentiation between $S$. divaricata and P. japonicum roots and between $A$. decursiva and P. praeruptorum roots $[17,18]$. However, in contrast with diverse genetic studies, no studies have investigated the chemotaxonomic relationship between $P$. japonicum, $S$. divaricata, P. praeruptorum, and A. decursiva. Moreover, the P. japonicum, S. divaricata, P. praeruptorum, and $A$. decursiva samples used in the aforementioned articles were limited in their chemical analysis and were not apparently authenticated at the species level.

The genetic authentication-based chromatographic profiling method is an emerging technique that is mainly performed by DNA-barcoding hyphenated chromatographic analysis. The combined method provides species-level accuracy of herbal samples, and therefore, a more reliable classification of those samples can be acquired by chemical analysis [19]. This technique has been applied to the chemical classification of diverse herbal medicines, such as Arnebia [20], Fritillaria [21], Phellodendron [22], and Daphne [23] species. Our group has also previously developed a 'genetic authentication-based chromatographic 
profiling' method for chemically distinguishing between Atractylodes species and Amomum species, using ITS sequencing, HPLC analysis, and chemometrics [24-26].

In this research, we collected samples of the aforementioned four species and confirmed their genetic authentication using ITS sequence-based DNA barcoding analysis to establish the chemotaxonomic relevance of $P$. japonicum (PJ), S. divaricata (SD), P. praeruptorum (PP), and A. decursiva (AD). Thereafter, chemical profiling of the samples was carried out using an HPLC-diode array detector (HPLC-DAD) and chemotaxonomic classification was performed by chemometric analysis.

\section{Results and Discussion}

\subsection{Internal Transcribed Spacer Regions of Nuclear Ribosomal Cistron}

The nucleotide sequences of the ITS (ITS1, ITS2, include $5.8 \mathrm{~s}$ ) were used for identification of the species of distributed Peucedani Radix, Peucedami Japonici radix and Saposhnikoviae Radix. Around 689-693 bp amplified nucleotide sequences were determined, based on the samples listed in Tables 1 and 2. The determined sequences were confirmed using the Blast in NCBI GenBank data. In total, 44 site differences were observed among the four species shown in Tables 1 and 2 (Table 3). A difference in 37 sites was observed between two species of Peucedani Radix (PJ and AD) with a 0.94 sequence identity matrix (Table S1). The two same genus samples, PP and PJ, had 16 site differences (sequence identity matrix 0.975; Table S1). SD and PJ had nine different sites (sequence identity matrix 0.986 ; Table S1). The results indicated that the ITS could be used as a clear and useful tool to identify the species of medicinal herbs in Tables 1 and 2.

Table 1. Species identification of the samples using the combined five DNA barcode analysis.

\begin{tabular}{|c|c|c|c|c|c|}
\hline Code & $\begin{array}{c}\text { Species } \\
\text { Identification }\end{array}$ & Geographic Origin & Code & $\begin{array}{c}\text { Species } \\
\text { Identification }\end{array}$ & Geographic Origin \\
\hline PJ-01 & $\begin{array}{l}\text { Peucedanum } \\
\text { japonicum }\end{array}$ & - & PP-15 & P. praeruptorum & China \\
\hline PJ-02 & P. japonicum & Yeongcheon, Gyeongbuk, Korea & PP-16 & P. praeruptorum & Zhejiang, China \\
\hline PJ-03 & P. japonicum & - & PP-17 & P. praeruptorum & Zhejiang, China \\
\hline PJ-04 & P. japonicum & Yeongju, Gyeongbuk, Korea & PP-18 & P. praeruptorum & Zhejiang, China \\
\hline PJ-05 & P. japonicum & 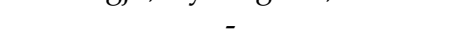 & PP-19 & P. praeruptorum & Zhejiang, China \\
\hline PJ-06 & P. japonicum & Gyeongbuk, Korea & PP-20 & P. praeruptorum & Zhejiang, China \\
\hline PJ-07 & P. japonicum & Gyeongbuk, Korea & PP-21 & P. praeruptorum & China \\
\hline PJ-08 & P. japonicum & Yeongju, Gyeongbuk, Korea & PP-22 & P. praeruptorum & - \\
\hline PJ-09 & P. japonicum & Gyeongbuk, Korea & PP-23 & P. praeruptorum & - \\
\hline PJ-10 & P. japonicum & Yeongju, Gyeongbuk, Korea & PP-24 & P. praeruptorum & - \\
\hline PJ-11 & P. japonicum & Korea & PP-25 & P. praeruptorum & China \\
\hline PJ-12 & P. japonicum & Hwasun, Jeonnam, Korea & PP-26 & P. praeruptorum & Yunnan, China \\
\hline PJ-13 & P. japonicum & Bonghwa, Gyeongbuk, Korea & PP-27 & P. praeruptorum & Zhejiang, China \\
\hline PJ-14 & P. japonicum & Yeongju, Gyeongbuk, Korea & AD-01 & Angelica decursiva & - \\
\hline PJ-15 & P. japonicum & Bonghwa, Gyeongbuk, Korea & AD-02 & A. decursiva & - \\
\hline PJ-16 & P. japonicum & Korea & AD-03 & A. decursiva & - \\
\hline PP-01 & P. praeruptorum & China & AD-04 & A. decursiva & - \\
\hline PP-02 & P. praeruptorum & China & AD-05 & A. decursiva & - \\
\hline PP-03 & P. praeruptorum & China & AD-06 & A. decursiva & - \\
\hline PP-04 & P. praeruptorum & China & AD-07 & A. decursiva & China \\
\hline PP-05 & P. praeruptorum & China & SD-01 & $\begin{array}{c}\text { Saposhnikovia } \\
\text { divaricata }\end{array}$ & Neimenggu, China \\
\hline PP-06 & P. praeruptorum & China & SD-02 & S. divaricata & Hebei, China \\
\hline PP-07 & P. praeruptorum & China & SD-03 & S. divaricata & China \\
\hline PP-08 & P. praeruptorum & China & SD-04 & S. divaricata & - \\
\hline PP-09 & P. praeruptorum & China & SD-05 & S. divaricata & Neimenggu, China \\
\hline
\end{tabular}


Table 1. Cont.

\begin{tabular}{cccccc}
\hline Code & $\begin{array}{c}\text { Species } \\
\text { Identification }\end{array}$ & Geographic Origin & Code & $\begin{array}{c}\text { Species } \\
\text { Identification }\end{array}$ & Geographic Origin \\
\hline PP-10 & P. praeruptorum & China & SD-06 & S. divaricata & Neimenggu, China \\
PP-11 & P. praeruptorum & China & SD-07 & S. divaricata & Neimenggu, China \\
PP-12 & P. praeruptorum & China & SD-08 & S. divaricata & Jilin, China \\
PP-13 & P. praeruptorum & China & SD-09 & S. divaricata & China \\
PP-14 & P. praeruptorum & China & SD-10 & S. divaricata & China \\
\hline
\end{tabular}

'-': unknown.

Table 2. List of standard reference samples used for the genetic identification in this study.

\begin{tabular}{|c|c|c|c|}
\hline No. & Accession Code & Scientific Name & Medicinal Name \\
\hline 1 & PR-PP01 & \multirow{5}{*}{ Peucedanum praeruptorum Dunn } & \multirow{10}{*}{ Peucedani Radix } \\
\hline 2 & PR-PP02 & & \\
\hline 3 & PR-PP03 & & \\
\hline 4 & PR-PP04 & & \\
\hline 5 & PR-PP05 & & \\
\hline 6 & PR-AD01 & \multirow{5}{*}{$\begin{array}{l}\text { Angelica decursiva (Miq.) Franch. et Sav. } \\
\text { (=Peucedanum decursivum Maxim.) }\end{array}$} & \\
\hline 7 & PR-AD02 & & \\
\hline 8 & PR-AD03 & & \\
\hline 9 & PR-AD04 & & \\
\hline 10 & PR-AD05 & & \\
\hline 11 & PJR-PJ01 & \multirow{5}{*}{ Peucedanum japonicum Thunberg } & \multirow{5}{*}{ Peucedani Japonici Radix } \\
\hline 12 & PJR-PJ02 & & \\
\hline 13 & PJR-PJ03 & & \\
\hline 14 & PJR-PJ04 & & \\
\hline 15 & PJR-PJ05 & & \\
\hline 16 & SR-SD01 & \multirow{5}{*}{ Saposhnikovia divaricata Schischkin } & \multirow{5}{*}{ Saposhnikoviae Radix } \\
\hline 17 & SR-SD02 & & \\
\hline 18 & SR-SD03 & & \\
\hline 19 & SR-SD04 & & \\
\hline 20 & SR-SD05 & & \\
\hline
\end{tabular}

Table 3. Amplicon size of plastid loci and nuclear barcode regions in species of Tables 1 and 2 samples and sequence characteristics, namely single and different multi-locus combinations.

\begin{tabular}{|c|c|c|c|c|c|c|}
\hline Barcode Target & $\begin{array}{l}\text { Amplicon } \\
\text { Size ( bp) }\end{array}$ & $\begin{array}{c}\text { Aligned } \\
\text { Length (bp) }\end{array}$ & $\begin{array}{l}\text { Conserved } \\
\text { Sites }\end{array}$ & $\begin{array}{l}\text { Variable } \\
\text { Sites }\end{array}$ & $\begin{array}{c}\text { Parsimony } \\
\text { Informative Sites }\end{array}$ & $\begin{array}{l}\text { Singleton } \\
\text { Site }\end{array}$ \\
\hline ITS & 700 & $689-693$ & 648 & 44 & 2 & 42 \\
\hline matk & 930 & 933 & 920 & 13 & 4 & 9 \\
\hline$r b c L$ & 670 & 670 & 664 & 6 & 1 & 5 \\
\hline psbA-trnH & 320 & $311-345$ & 323 & 17 & none & 16 \\
\hline trnL-F intergenic sapcer & 440 & 444 & 440 & 4 & none & 4 \\
\hline $\begin{array}{l}\text { matk }+r b c L \\
p s b A-t r n H+\end{array}$ & & 1603 & 1584 & 19 & 5 & 14 \\
\hline trnL-F intergenic spacer & & $755-789$ & 763 & 21 & none & 20 \\
\hline$m a r k+r b c L+p s b A-t r n H$ & & 1914-1948 & 1907 & 36 & 5 & 30 \\
\hline $\begin{array}{c}\text { Mark }+r b c L+ \\
\text { trnL-F intergenic spacer }\end{array}$ & & 2047 & 2024 & 23 & 5 & 18 \\
\hline $\begin{array}{l}\text { Four plastid } \\
\text { targets }\end{array}$ & & 2358-2392 & 2347 & 40 & 5 & 34 \\
\hline
\end{tabular}




\subsection{Chloroplast Genome-Based DNA Barcode Sequence Analysis}

Four chloroplast DNA barcode regions $(r b c L$, marK, $p s b A-\operatorname{trn} H$, and $t r n L-F$ intergenic spacer) were analyzed to supplement the results of the ITS. The $p s b A-t r n H$ region had the most variable sites among the four plastid barcode regions (except the ITS region). On the other hand, the trnL-F intergenic spacer was the most conserved region among all of the analyzed DNA barcode regions (including ITS).

Although four analyzed plastid loci had less abundant variable sites and were highly conserved compared to the ITS region, it could be useful to separate the species used in this study.

To obtain more detail, a 390F/1326R primer set was used to amplify the mat $K$, and the 933-base partial nucleotide sequences were determined. The total number of variable sites was 13; however, some of them were highly conserved. In particular, there were only two site differences between PP and PJ. Nevertheless, matK could distinguish four species sufficiently.

For the $r b c L$ with a $r b c L$ a-f/724R primer set, 670-base partial nucleotide sequences were determined in all samples listed in Tables 1 and 2. Six variable sites were found, which was a well-conserved region among the five DNA barcode regions. The sequence identity matrix of $r b c L$ was $0.992-1.000$. In the $r b c L$ region, PP and PJ showed identical nucleotide sequences (sequence identity matrix 1, Supplementary Materials Table S1). Except for this case, other species could be identified by comparing $r b c L$ nucleotide sequences.

The $p s b A-t r n H$ with a $t r n H 2 / p s b A F$ primer set showed different lengths of amplified product dependent on the species, and around 311-345 base partial nucleotide sequences were determined. The $p s b A$-trnH had the shortest aligned length among the five DNA barcode regions but showed the most variable sites (17) among the four chloroplast barcode regions, and had several indel sequences. Therefore, the sequence identity matrix ranged from 0.904 to 0.944. Between PP and PJ, the closest result was 0.944 .

A trnL-e/trnL-f primer set was used for the trnL-F intergenic spacer, and 444-base nucleotide sequences were identified. As mentioned earlier, the trnL-F intergenic spacer was the most conserved region, and the sequence identity matrix ranged from 0.99 to 1.00 . Four variable sites were observed in $\mathrm{AD}$ alone, and the other three species showed the same sequence.

A single region chloroplast DNA barcode analysis approach and be inaccurate in determining the species' identity. Therefore, the Consortium for the Barcode of Life (CBOL)-Plant working group also recommends two- or more locus combinations to initiate the barcode process for plant species. The recommended standard combination locus is rbcL-matK, because this combination is a practical solution to the complex balance between universality, sequence quality classification, and cost. [27]. However, this combination is not always efficient and, in this case, three- or more locus analyses are necessary. Therefore, the $p s b A-t r n H$ and $t r n L-F$ intergenic spacer regions were additionally selected for analysis in this study. Both loci were quite short, which suggests that they could practically be used for the analysis of processed distributed samples. Unfortunately, the trnL-F intergenic spacer regions of the four species shown in Tables 1 and 2 were highly conserved. In contrast, the $p s b A$-trnH is already known as one of the most variable genome segments in the angiosperm chloroplasts. As expected, it had most variable sites in this study, but it also had many indel sequences. Therefore, single-locus analysis was not efficient.

\subsection{Phylogenetic Analysis}

To analyze the genetic relationship among the four species used in this study, the PhyML + SMS (Maximum likelihood-based inference of phylogenetic trees with Smart Model Selection) program was performed with concatenated nucleotide sequences of ITS and four chloroplast DNA barcodes. Four species commonly located in the Selineae tribe of the Apiacae family are clustered into two groups, genus Angelica and genus Peucedanum (including genus Saposhnikovia) (Figure 1). The results of genetic flexibility analysis of the four species show that PP and PJ are very close. Comparing the results with the plants of 
genus Peucedanum, PP and PJ, shows that they are not only the same genus, but genetically closer to other species. AD is located in the genus Angelica group and is relatively far in genetic distance compared to the other three species.

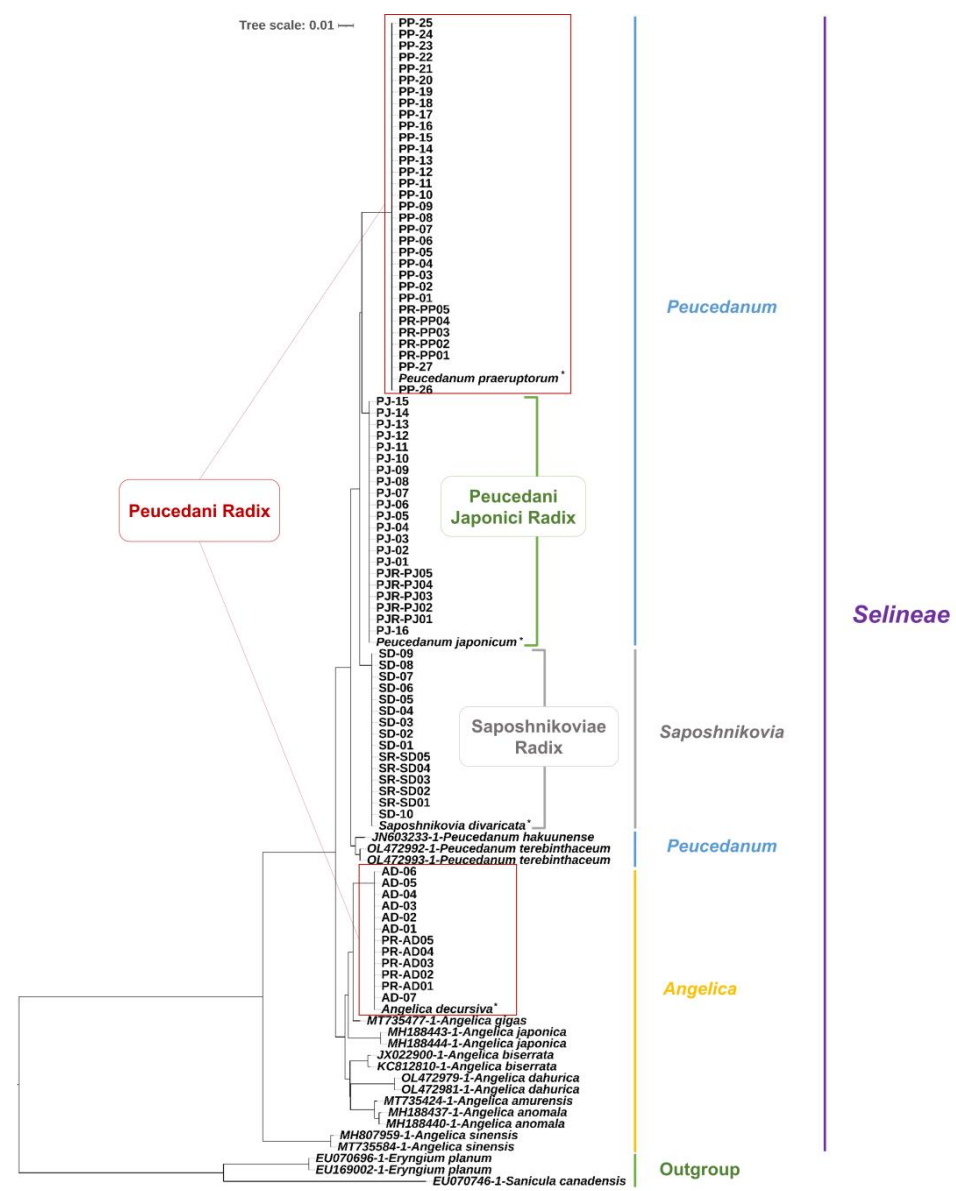

Figure 1. Maximum likelihood-based inference of phylogenetic tree with Smart Model Selection, constructed based on the combined five DNA barcode regions (ITS and four plastids). PJ: Peucedanum japonicum, PP: P. praeruptorum, AD: Angelica decursiva, SD: Saposhnikovia divaricata. ‘*' represents the NCBI Genbank data combination.

\subsection{Chromatographic Profiling of PJ, PP, AD, and SD Samples}

HPLC analytical conditions, including the mobile phase modifier, mobile phase composition, and UV detection wavelength, were optimized for the chromatographic analysis of the samples. The addition of $0.1 \%$ trifluoroacetic acid (TFA; $v / v$ ) in water with acetonitrile was chosen owing to better inter-peak separation and clearer detection of peaks under the acidic mobile phase in the mobile phase without TFA. The UV detection wavelengths were selected based on the optimal absorbance of each peak as follows: 4 peaks at UV $235 \mathrm{~nm}, 13$ peaks at UV $250 \mathrm{~nm}, 14$ peaks at UV $275 \mathrm{~nm}, 4$ peaks at UV $300 \mathrm{~nm}, 10$ peaks at UV $310 \mathrm{~nm}, 46$ peaks at UV $325 \mathrm{~nm}, 7$ peaks at UV $323 \mathrm{~nm}$, and 2 peaks at UV $350 \mathrm{~nm}$ (Supplementary Materials Table S2).

The intraday precision of the sample was $<0.2 \%$ for retention time and $<4.0 \%$ for the absolute peak area, and interday precision was $<0.1 \%$ for retention time and $<3.0 \%$ for the peak area (Supplementary Materials Table S3).

The overlapping intra-species chromatograms showed similar patterns within the samples of each species, except for a few outliers in the AD and SD samples, whereas those of inter-species comparisons showed slightly different patterns among PJ, PP, and AD samples, with a few identical peaks for PJ and AD samples after a retention time of 
$40 \mathrm{~min}$. The chromatographic patterns of the SD samples were distinguishable from those of the PJ, PP, and AD samples over the entire retention time (Figures 2 and S1).
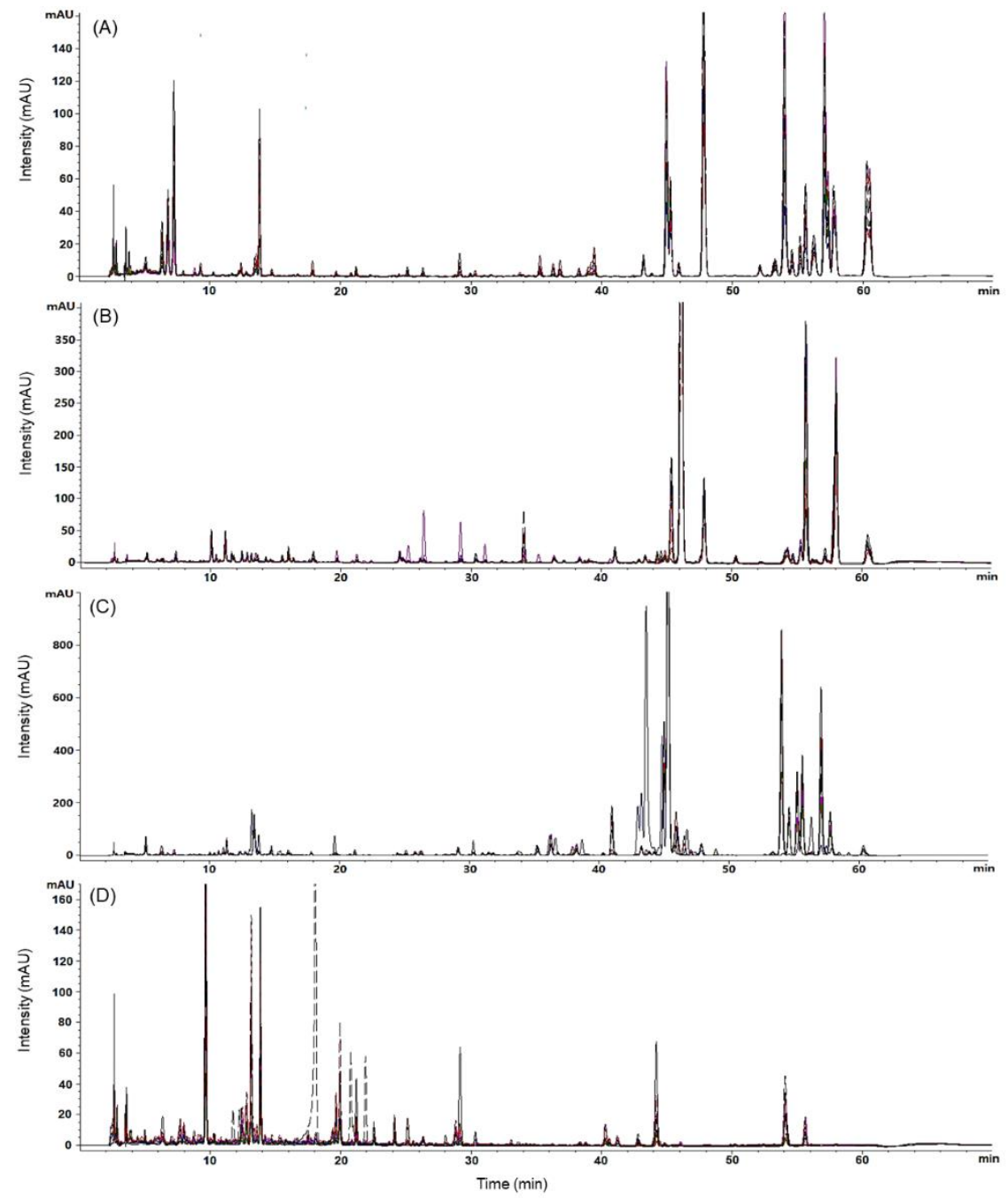

Figure 2. Overlapping chromatograms of representative samples of Peucedanum japonicum ((A) PJ0110), P. praeruptorum ((B) PP01-10), Angelica decursiva ((C) AD01-07), and Saposhnikovia divaricata ((D) SD01-10) at a detection wavelength of $325 \mathrm{~nm}$.

Among the peaks commonly occurring in more than two species, the average peak areas of many peaks were significantly different between two species as follows: 26 peaks between PJ and AD samples (peaks 1, 3, 4, 14, 17, 18, 23, 33, 46, 47, 49, 52, 53, 54, 64, 69, 70, 76, 88, 90, 91, 92, 95, 97, 99, and 100); 15 peaks between PJ and PP samples (peaks 2, $4,6,14,17,18,23,39,70,76,88,92,93,95$, and 100); 4 peaks between PJ and SD samples (peaks 2, 18, 36, and 45); 27 peaks between PP and AD samples (peaks 1, 6, 11, 12, 13, 17, 23, $39,49,53,61,64,70,76,79,81,82,83,84,85,87,88,90,91,93,94,95$, and 100); 17 peaks between PP and SD samples (peaks $6,12,15,17,18,22,36,45,57,61,62,66,73,81,83,85$, and 92); 10 peaks between AD and SD samples (peaks 18, 45, 56, 79, 81, 82, 83, 84, 85, and 92) (Figure S2).

\subsection{Clustering Analysis of the Samples Using Chemometric Statistical Methods}

The chemotaxonomic correlations between PJ samples and PP, AD, and SD samples were measured using chemometric clustering tools, principal component analysis, and $k$-means clustering analysis. As shown in Figure 3, the samples of each species formed distinct clusters based on their own origins in the principal component (PC) scores, PC1 
and PC2, which explained $21.7 \%$ and $18.7 \%$ of the total variance, respectively. PJ, PP, and AD samples were densely located in their own species groups which were clearly separated from each other. Although the SD samples also formed clusters, they were distributed widely in the SD group, resulting in the interruption of a few samples (SD02 and SD03) in the PP group. The PJ samples were located closer to the PP samples than to the SD samples, whereas the AD samples were exceptionally different from the other sample groups in terms of PC1 and PC2 scores.

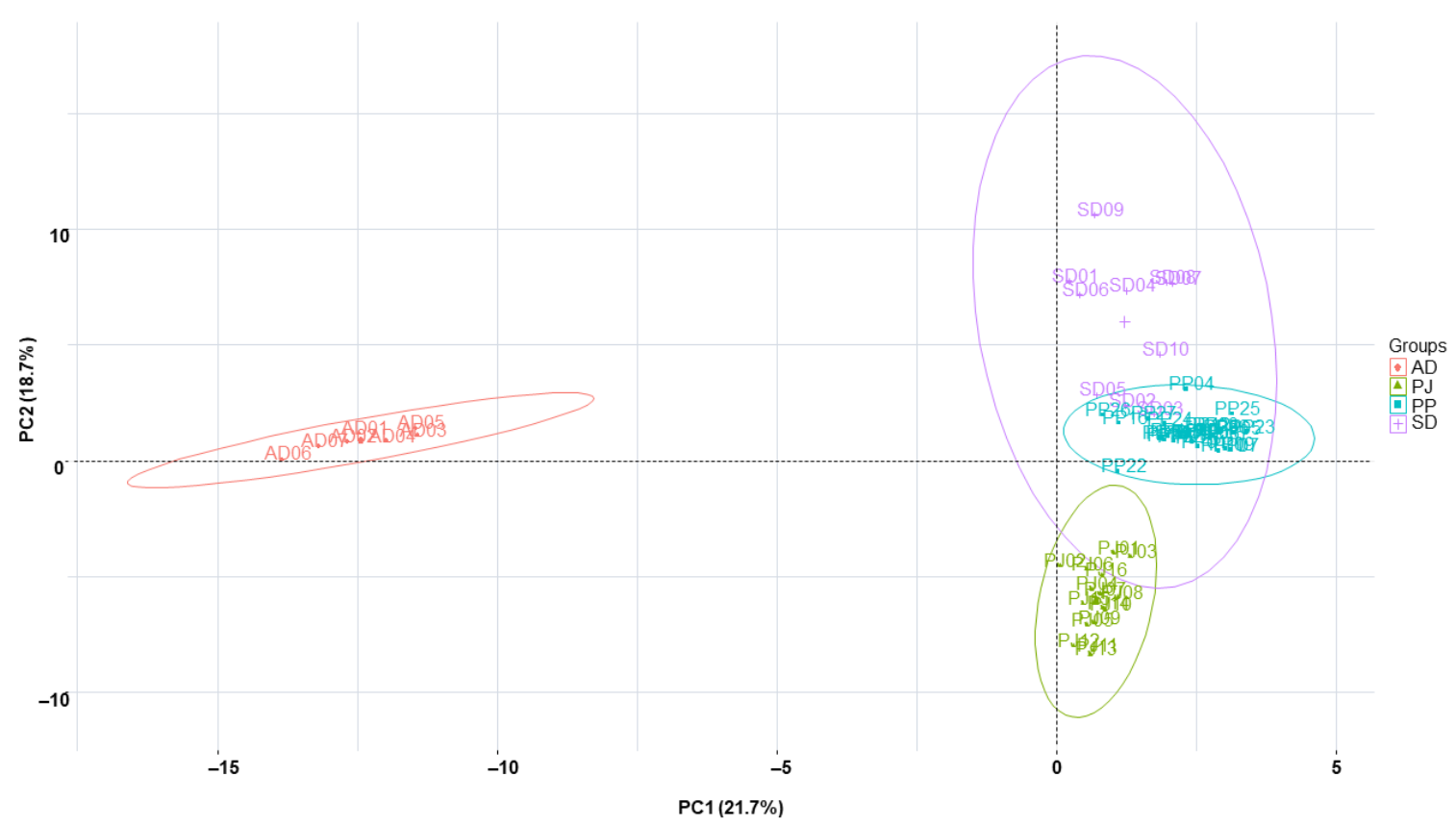

Figure 3. Score plot of principal components (PC1 vs. PC2) based on the variables (absolute area of reference peaks) with Peucedanum japonica, P. praeruptorum, Angelica decursiva, and Saposhnikovia divaricata samples based on a 99\% confidence ellipse. PC1 and PC2 represent 21.7\% and $18.7 \%$ of the total variance, respectively. PJ: Peucedanum japonicum, PP: P. praeruptorum, AD: Angelica decursiva, SD: Saposhnikovia divaricata.

In the $k$-means clustering analysis, four clusters were selected as the optimal number of clusters, which was determined using the silhouette method (Figure S3). The distribution of samples according to their dimension scores was consistent with those in the PC plot. However, the grouping of samples was different, as follows: PP01, PP26, SD02, and SD05 samples were contained in the PJ cluster and PP27 was contained in the SD cluster. Hence, the PJ cluster was intruded by the PP cluster which also slightly overlapped with the SD cluster. The AD cluster also showed a distinct distance from the other clusters, as shown in the PC plot (Figure 4). 


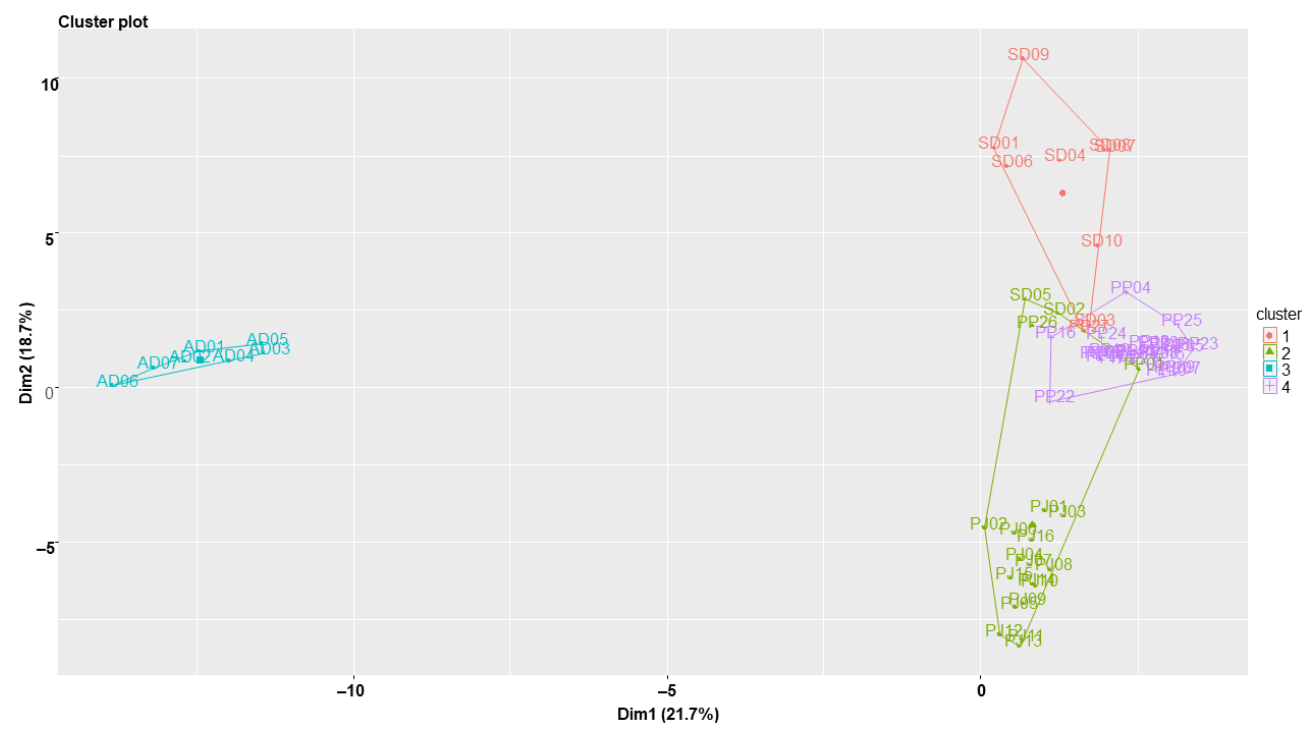

Figure 4. $k$-Means clustering plot of Peucedanum japonica, P. praeruptorum, Angelica decursiva, and Saposhnikovia divaricata samples. Dim: dimension. PJ: Peucedanum japonicum, PP: P. praeruptorum, AD: Angelica decursiva, SD: Saposhnikovia divaricata.

The results of clustering analyses demonstrate that the PJ samples showed better chemical proximity with the PP samples than the SD samples due to the PC (=dimension score in $k$-means) scores of the PJ samples being closer to those of the PP samples than the SD samples $[28,29]$.

\subsection{Evaluation of Similarity between the Samples Using Pearson's Correlation Coefficient}

The similarity among the PJ, PP, AD, and SD samples was evaluated using Pearson's correlation coefficient $(r)$, which ranged from -1 to +1 (Table S4). In the correlation plot, the PJ samples showed a higher correlation with the AD samples $(r=0.791$ for mean and 0.976 for median), followed by most PP samples ( $r=0.029$ for mean and 0.021 for median), and were negatively correlated with the SD samples $(r=-0.099$ for mean and -0.097 for median). The correlations between the PP and AD samples ( $r=-0.011$ for mean and -0.014 for median), PP and SD samples ( $r=-0.038$ for mean and median), and AD and SD samples ( $r=-0.061$ for mean and -0.060 for median) were not different. The mean and median $r$ values also showed that the intra-species relationships were as follows: $\mathrm{PJ}-\mathrm{PJ}>\mathrm{PP}-\mathrm{PP}=\mathrm{AD}-\mathrm{AD}>\mathrm{SD}-\mathrm{SD}$. Meanwhile, inter-species relationships were as follows: PJ-AD > PJ-PP > PP-AD > PP-SD > AD $\times$ SD > PJ-SD (Figure 5 and Table 4). 


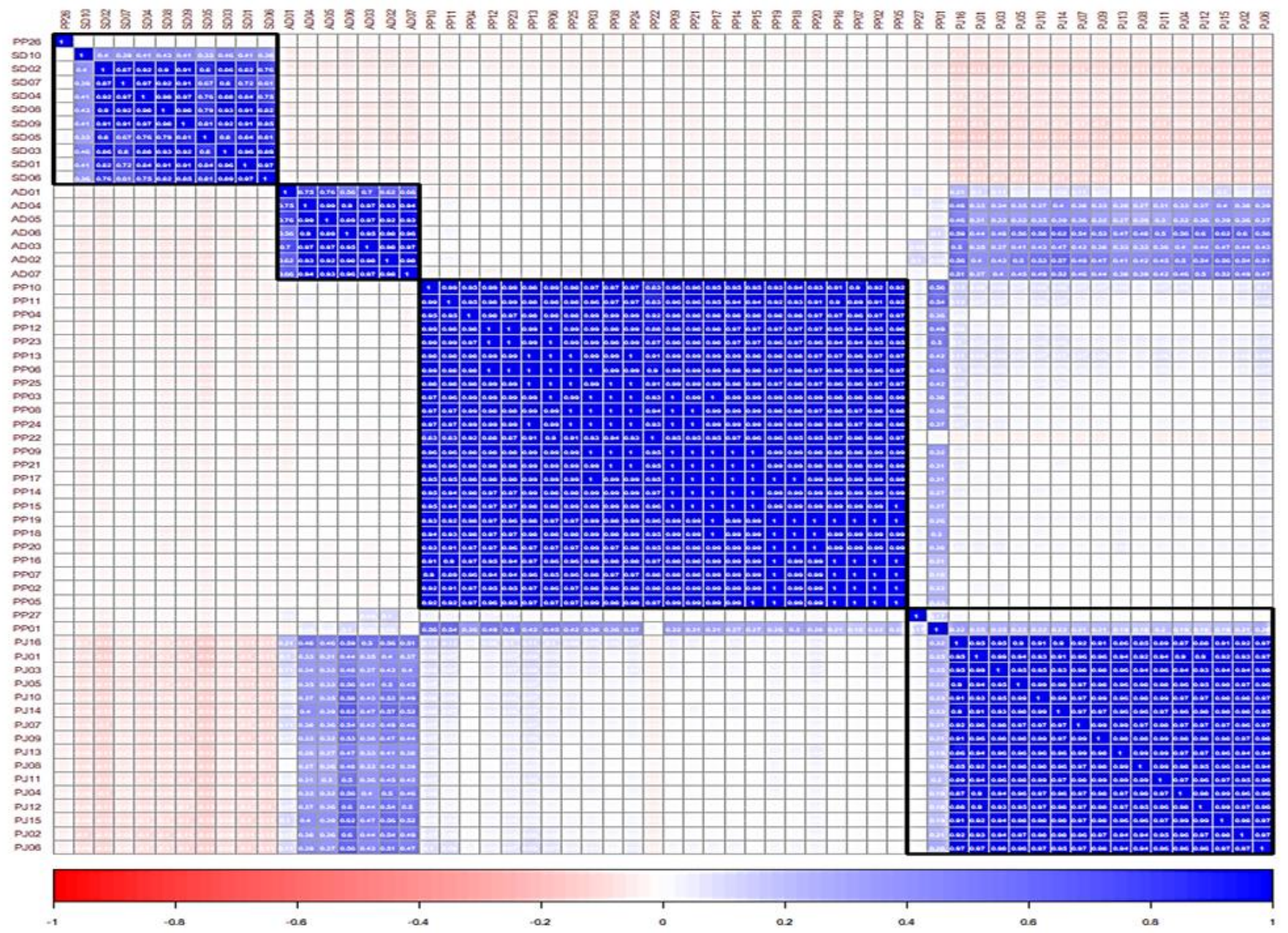

Figure 5. Correlation plot of the Pearson's correlation coefficient $(r)$ among Peucedanum japonica, P. praeruptorum, Angelica decursiva, and Saposhnikovia divaricata samples. The range of the coefficient is -1 (red color) $<r<1$ (blue color). PJ: Peucedanum japonicum, PP: P. praeruptorum, AD: Angelica decursiva, SD: Saposhnikovia divaricata.

Table 4. Pearson's correlation coefficients among PJ, PP, AD, and SD samples.

\begin{tabular}{cccccc}
\hline Sample & Value & PJ & PP & AD & SD \\
\hline \multirow{4}{*}{ PJ } & Mean & 0.957 & & & \\
& Median & 0.966 & & & \\
& Max & 0.991 & & & \\
Min & 0.849 & & & \\
PP & Mean & 0.029 & 0.791 & & \\
& Median & 0.021 & 0.976 & & \\
& Max & 0.321 & 0.999 & & \\
AD & Min & -0.058 & -0.034 & & \\
& Mean & 0.791 & -0.011 & 0.872 & 0.932 \\
& Median & 0.976 & -0.014 & 0.995 & 0.822 \\
& Max & 0.999 & 0.103 & 0.563 & 0.982 \\
& Min & -0.034 & -0.057 & -0.061 & \\
\hline
\end{tabular}

PJ: Peucedanum japonicum, PP: P. praeruptorum, AD: Angelica decursiva, SD: Saposhnikovia divaricata. 
The average $r$ values of the samples of each species relative to the individual samples of other independent species exhibited diverse intra- and inter-sample variations compared to the rest of the species samples, that is, higher correlations for PP01-PJ samples, PP01- and PP27-AD samples, and PP26-SD samples and lower correlation for AD01-PJ samples, PP01PP samples, AD01-PP samples, AD01-AD samples, and SD10-SD samples (Figures S4-S7).

Positive and higher coefficient $(r)$ values between the PJ and the AD samples indicate a stronger inter-species correlation, as compared with the weaker inter-species correlation between the PJ and the PP samples, with lower $r$ values. Negative and weaker $r$ values between the PJ and the SD samples indicated that their inter-species similarity was lower than those with positive $r$ values [30,31].

The chemotaxonomic classification of PJ, PP, AD, and SD samples based on phylogenetic authentication was successfully evaluated using chromatographic profiling combined with chemometric analysis, and their chemical characteristics were clearly reflected by their own species. The chemical correlation between PJ and the other three species was also investigated, and it was observed that PJ had a strong chemical relationship with PP and $\mathrm{AD}$, depending on the measured chemometric analyses; however, its chemical relationship with SD was less or even negligible.

As the therapeutic activity of herbal medicines can possibly be explained by their chemical constituents, the chemotaxonomic closeness between PJ and PP or AD might provide evidence for their analogous medicinal application to reduce phlegm and heatinduced respiratory symptoms. Meanwhile, the chemotaxonomic distance between PJ and SD presumably indicates their therapeutic dissimilarity with respect to relieving common cold-induced disorders. Further pharmacological and clinical studies are required to confirm these chemotaxonomic results.

Nonetheless, there are a few limitations to this study, as follows: (1) sample numbers for AD or SD were insufficient, mainly owing to difficulties in collecting genuine species; (2) intra-species chemical variation in SD samples was prominent, presumably owing to diverse habitats [32,33], and this could be caused by environmental factors, including temperature, dryness/humidity, rainfall, soil conditions, and altitude, which definitely affect the production of secondary metabolites in herbal plants [34-36]; (3) insufficient research on chemical classifications among the aforementioned four species.

\section{Materials and Methods}

\subsection{Plant Materials and Reagents}

Methanol, water, and acetonitrile (HPLC grade) were purchased from J. T. Baker (Phillipsburg, NJ, USA). TFA was purchased from Sigma-Aldrich (St. Louis, MO, USA). Prim-O-glucosyl-cimifugin (peak 10), cimifugin (peak 19), ubelliferone (peak 21), secO-glucosyl-hamaudol (peak 34), psoralen (peak 42), xanthotoxin (peak 44), bergapten (peak 45), oxypeucedanin (peak 48), imperatorin (peak 57), decursin (peak 66), praeruptorin A (peak 73), praeruptorin B (peak 92), and praeruptorin C (peak 98) were purchased from ChemFaces (Wuhan, China).

Sixteen samples of PJ, twenty-seven samples of PP, seven samples of AD, and ten samples of SD were collected from agricultural plantations, natural habitats, and markets in Korea and China, and were also supplied by the Korea Institute of Oriental Medicine (Table 1). The collected samples were morphologically authenticated by the authors (J.H. Kim and G. Lee). For genetic identification, 20 additional voucher samples were used as standard reference samples (Table 2). All voucher specimens (code No. PNUKM-2021PJ01-PJ16, PP01-PP27, AD01-AD07, and SD01-SD10) and extracted genomic DNA were deposited at the herbarium of the College of Korean Medicine in Wonkwang University and at the School of Korean Medicine, Pusan National University.

\subsection{Preparation of Genomic DNA}

A NucleoSpin ${ }^{\circledR}$ Plant II kit (Macherey-Nagel, Dueren, Germany) with PL1 lysis buffer was used to extract the genomic DNA of the samples in Tables 1 and 2. Some samples 
required extra steps to treat $10 \%$ cetyltrimethylammonium bromide with $0.7 \mathrm{M} \mathrm{NaCl}$ added to remove high levels of phenolic compounds and/or polysaccharides.

\subsection{PCR Amplification for DNA Barcode Analysis}

For DNA barcode analysis, PCR amplification was performed using a T-personal cycler (Biometra, Jena, Germany). Briefly, 1X AccuPower ${ }^{\circledR}$ GoldHotStart Taq PCR PreMix (Bioneer, Daejeon, Korea) with $600 \mathrm{nM}$ primer set and $30 \mathrm{ng}$ of genomic DNA were used for each PCR amplification. The primer set ITS1 (5'-TCCGTAGGTGAACCTGCGG-3') and ITS4 $\left(5^{\prime}\right.$-TCCTCCGCTTATTGATATGC-3') [37] were used to amplify the ITS include 5.8 s. For the chloroplast DNA barcodes, $r b c L$ a-f (5'-ATGTCACCACAAACAGAGACTAAAGC-3') /724R (5'-TCGCATGTACCTGCAGTAGC-3') and 390F (5'-CGATCTATTCA TTCAATATTTC$\left.3^{\prime}\right)$ /1326R (5'-TCTAGCACACGAAAAGTCGAAGT-3') primer sets were used for $r b c L$ and matK amplification, respectively [38-40]. For the $t r n L-F$ intergenic spacer, the $t r n L-e$ (5'-GGTTCAAGTCCCTCTTATCCC-3')/trnL-f (5'-ATTTGAACTGGTGACACGAG-3') set and the primer set $t r n H 2$ (5'-CGCGCATGGTGGATTCACAATCC-3')/ $p s b A F\left(5^{\prime}\right.$-GTTATGCATG AACGTAATGCTC-3') were used for the $p s b A$-trnH regions $[27,41]$. The amplified products were separated and confirmed by electrophoresis by using $1.5 \%$ agarose gel added with Safe-View ${ }^{\mathrm{TM}}$ (abm, Richmond, VA, Canada).

\subsection{Determination of DNA Sequences of PCR Product}

All of the PCR products were sub-cloned after being separated from agarose gels by use of a TOPcloner ${ }^{\mathrm{TM}}$ TA Kit (Enzynomics, Daejeon, Korea). The DNA sequences were then determined through an interpretation performed by Bioneer (Daejeon, Korea). To improve the accuracy of the results, the DNA barcode analysis was repeated three times, independently from the genomic DNA extraction stage.

\subsection{Analysis of DNA Sequences and Preparation of Dendrogram}

The determined DNA sequences were first analyzed using Bioedit's ClustalW multiple sequence alignment (Bioedit, v7.0.9; available from http: / / www.mbio.ncsu.edu/BioEdit/ page2.html, accessed on 28 November 2021) and reconfirmed with multiple sequence alignment using MAFFT (MAFFT, v7; available from https:/ / mafft.cbrc.jp/alignment/server/, accessed on 29 November 2021) [42]. To confirm the polymorphism represented by IUPAC symbols, all sequences were identified at least twice using a chromatogram of nucleotide sequences provided by the Bioneer sequencing service. The phylogenetic tree for the ITS region was analyzed using MAFFT (multiple alignment, v7.407_1), BMGE (alignment curation, v.1.12_1) [43] and PhyML (tree inference based on the maximum-likelihood, v.3.1_1) [44,45] workflow (PhyML/OneClick, available from https://ngphylogeny.fr/, accessed on 3 December 2021). Phylogenetic analysis of the combined five DNA barcodes (ITS and four plastid regions) was followed by the PhyML+SMS/OneClick method, which was performed in accordance with MAFFT, BMGE, and PhyML+SMS (maximum likelihood-based inference of phylogenetic trees with Smart Model Selection, available from https://ngphylogeny.fr/, accessed on 8 December 2021) [46]. All sequence results completed in the analysis were compared and confirmed with the NCBI GenBank using Blast [47]. The reference data ITS nucleotide sequences for analysis of the genetic relationships comes from NCBI Genbank and were represented by the accession number in phylogenetic tree. The Chloroplast barcode data were also collected from NCBI Genbank, including MT671397.1, NC033344.1, OL362112.1, MW436378.1, MW820164.1, MW900177.1, MT921980.1, MT921.997, and KX352468.1. Two species, Eryngium planum (EU169002, EU0706969, MT561039.1) and Sanicula canadensis (EU070746.1, KP642834.1, KP643255.1, KJ773865.1), were used as the outgroup [48].

\subsection{Preparation of Samples for HPLC Analysis}

The pulverized PJ, SD, PP, and AD samples were homogenized using a $500 \mu \mathrm{m}$ testing sieve (Chunggyesanggong-sa; Gunpo, Gyeonggi, Korea). The extraction was performed 
using a powder of $500 \mathrm{mg}$ with $5 \mathrm{~mL}$ of methanol using an ultrasonic extractor (Power Sonic 520; Hwashin Tech, Daegu, Korea) for $30 \mathrm{~min}$. The extract, filtered through a $0.2 \mu \mathrm{m}$ syringe filter (BioFact, Daejeon, Korea), was evaporated using a nitrogen-blowing concentrator (MGS2200; Eyela, Miyagi, Japan) and was re-dissolved in HPLC-grade methanol at a concentration of $20,000 \mu \mathrm{g} / \mathrm{mL}$ prior to HPLC injection.

\subsection{HPLC Conditions for Chromatographic Profiling}

Chromatographic analysis was performed using an Agilent 1200 liquid chromatography system (Agilent Technologies; Palo Alto, CA, USA) consisting of an autosampler, degasser, solvent pump, and DAD. Data were processed using ChemStation (Agilent Technologies). The chemical constituents, including 13 marker compounds, were separated on a Capcell Pak Mg II C 18 column (4.6 mm $\times 250 \mathrm{~mm}, 5 \mu \mathrm{m}$; Shiseido, Tokyo, Japan) at $35{ }^{\circ} \mathrm{C}$ with $1 \mathrm{~mL} / \mathrm{min}$ flow rate and $10 \mu \mathrm{L}$ injection volume. The mobile phase consisted of water containing $0.1 \%$ TFA (A) and acetonitrile (B), with the following gradient elution: $15 \%$ (B) over $0-2 \mathrm{~min}, 15-50 \%$ (B) over $2-30 \mathrm{~min}, 50 \%$ (B) over 30-32 $\mathrm{min}, 50-75 \%$ (B) over $32-55 \mathrm{~min}, 75 \%$ (B) over $55-58 \mathrm{~min}$, and then re-equilibrated to $15 \%$ (B) until the end of the analysis. Detection was performed using a UV detector at wavelengths of 235, 250, 275, $300,310,325,335$, and $350 \mathrm{~nm}$.

The precision of the analytical methods was determined by analyzing the retention times and absolute areas of selected peaks of PJ samples within one day (intraday precision) and over three consecutive days (interday precision). Precision was represented as relative standard deviations $($ RSDs $)$, where RSD $(\%)=(($ standard deviation/mean value $) \times 100)$.

\subsection{Chemometric Statistical Analysis}

The genetically identified samples of PJ, PP, AD, and SD were analyzed using HPLC, and the chemical relationship between the samples was evaluated using chemometric tools, that is, principal component analysis, $k$-means cluster analysis, and Pearson's correlation analysis. In total, 100 peaks were selected as profiling peaks, which were $>1.0 \%$ of the total peak area. Sixty samples and the absolute area of each profiling peak were used as a matrix for construction of the PC plot, the $k$-means cluster plot, and for the calculation of Pearson's correlation coefficient. The difference between the absolute area of each peak from the samples of independent species was evaluated using Tukey's test, with significance at $p<0.05, p<0.01$, and $p<0.001$. Chemometric analyses and Tukey's test were conducted using the open source software R (v. 4.1.2; The R Foundation for Statistical Computing).

\section{Conclusions}

Overall, 60 samples of PJ, PP, AD, and SD were phylogenetically authenticated using ITS and chloroplast genome-based DNA barcoding analysis at the species level. Chemotaxonomic classification of PJ and its chemical correlation with the remaining three species were investigated using chromatographic profiling with chemometric analyses. PJ samples, which showed the closest phylogenetic relationship with PP samples, showed a stronger chemical correlation with PP or AD samples but a weaker or even negative chemical correlation with SD samples. The results from the phylogenetic analysis-hyphenated chemotaxonomic correlation suggested the transfer of PJ from SD to the category of PP or AD for medicinal applications. Further pharmacological and clinical study would be necessary to support the chemical re-categorization of PJ.

Supplementary Materials: The following supporting information can be downloaded online, Figure S1: Chromatograms of the samples of Peucedanum japonicum, P. praeruptorum, Angelica decursiva, and Saposhnikovia divaricata; Figure S2: Multiple comparison of absolute peak areas among the samples; Figure S3: Silhouette plot; Figures S4-S7: Average Pearson's correlation coefficients among the samples; Table S1: Sequence identity matrix; Table S2: Retention times and detection wavelengths of profiling peaks; Table S3: Precision of profiling peaks; Table S4: Pearson's correlation coefficients among the samples. 


\begin{abstract}
Author Contributions: Conceptualization, J.-H.K., E.-J.D. and G.L.; data curation, J.-H.K., E.-J.D. and G.L.; methodology, J.-H.K., E.-J.D. and G.L.; supervision, G.L.; visualization, J.-H.K. and E.-J.D.; writing—original draft, J.-H.K. and E.-J.D.; writing—review and editing, J.-H.K., E.-J.D. and G.L. All authors have read and agreed to the published version of the manuscript.
\end{abstract}

Funding: This paper was supported by Wonkwang University in 2021.

Institutional Review Board Statement: Not applicable.

Informed Consent Statement: Not applicable.

Data Availability Statement: Data sharing not applicable.

Acknowledgments: The authors thank the Korean Herbarium of Standard Herbal Resources, Korea Institute of Oriental Medicine for providing the sample materials.

Conflicts of Interest: The authors declare no conflict of interest.

Sample Availability: Samples are not available from the authors.

\title{
References
}

1. The Ministry of Korean Food and Drug Safety. The Korean pharmacopoeia 12th edition. In KFDA Notification No. 2019-102; Ministry of Korean Food and Drug Safety: Cheongju-si, Korea, 2019.

2. Commission of Chinese Pharmacopeia. Pharmacopoeia of the Peoples Republic of China; China Medical Science Press: Beijing, China, 2015.

3. The Ministry of Korean Food and Drug Safety. The Korean herbal pharmacopoeia. In KFDA Notification No. 2020-73; Ministry of Korean Food and Drug Safety: Cheongju-si, Korea, 2020.

4. Peucedanum japonicum Thunberg in Murray. The Flora of China, Volume 14, p. 186. Available online: http://www.efloras.org/ florataxon.aspx?flora_id=2\&taxon_id=200015732 (accessed on 7 December 2021).

5. Editing Commission for Chinese Materia Medica, National Chinese Medicine and Pharmacy Administration. Chinese Materia Medica; Shanghai Science and Technology Press: Shanghai, China, 1999; Volume 5.

6. Kalpana, J.; Preeti, C.; Dnyaneshwar, W.; Bhushan, P. Molecular markers in herbal drug technology. Curr. Sci. 2004, 87, 159-165.

7. Choi, H.Y.; Seo, Y.B.; Lee, S.I. PCR-mediated RFLP to identify ‘Bangpoong' a crude drug. Kor. J. Pharmacogn. 1997, $28,1-8$.

8. Hong, S.M.; Lee, M.Y.; Koh, J.C.; Ko, B.S. Development and application of PCR-based markers for the discrimination of Bang-Poong and related species. Kor. J. Plant Biotechnol. 2004, 31, 1-6.

9. Cho, J.H.; Lim, J.M.; Kim, M.S.; Byeon, J.H.; Park, H.S.; Ahn, Y.S.; Park, C.G. Classification and discrimination of geographical origin of Bang-Poong (Saposhnikovia divaricata (Turcz) Schischkin) medicinal plant and related species by using DNA sequence analysis. Korean J. Intl. Agri. 2013, 25, 395-405.

10. Lee, M.Y.; Ju, Y.S.; Kim, H.J.; Ko, B.S. Morphological characteristic and PCR analysis for original identification of Peucedanum decursivum. Korean J. Ori. Med. 2003, 9, 113-122. [CrossRef]

11. Xiong, Y.X.; Wu, L.; Liu, Y.M.; Chen, K.L.; Xiong, Y.X.; Wu, L.; Liu, Y.M.; Chen, K.L. Identification of Peucedani Radix and its adulterants by DNA barcoding technique. Zhong Yao Cai 2013, 36, 1762-1765.

12. Hou, D.Y.; Song, J.Y.; Yang, P.; Zhou, H.; Xin, T.Y.; Yao, H. Identification of peucedani radix, peucedani decursivi radix and its adulterants using ITS2 sequence. Zhongguo Zhong Yao Za Zhi 2014, 39, 4186-4190.

13. Choi, S.A.; Kim, Y.J.; Lee, W.K.; Kim, K.Y.; Kim, J.H.; Seong, R.S. The complete chloroplast genome of the medicinal plant Angelica decursiva (Apiaceae) in Peucedani Radix. Mitochondrial DNA B Resour. 2016, 1, 210-211. [CrossRef] [PubMed]

14. Wang, L.; Yu, X.; Xu, W.; Zhang, J.; Lin, H.; Zhao, Y. Complete chloroplast genome sequencing support Angelica decursiva is an independent species from Peucedanum praeruptorum. Physiol. Mol. Biol. Plants 2021, 27, 2503-2515. [CrossRef] [PubMed]

15. Choi, B.R.; Yoon, D.; Kim, G.S.; Han, K.S.; Choi, D.J.; Lee, Y.S.; Hyun, D.Y.; Lee, D.Y. Metabolic profiling and method validation of marker compounds from Saposhnikoviae Radix and Peucedani Japonici Radix. J. Appl. Biol. Chem. 2020, 63, 393-399. [CrossRef]

16. Yoshitomi, T.; Wakana, D.; Uchiyama, N.; Tsujimoto, T.; Kawano, N.; Yokokura, T.; Yamamoto, Y.; Fuchino, H.; Hakamatsuka, T.; Komatsu, K.; et al. Identifying the compounds that can distinguish between Saposhnikovia root and its substitute, Peucedanum ledebourielloides root, using LC-HR/MS metabolomics. J. Nat. Med. 2020, 74, 550-560. [CrossRef] [PubMed]

17. Seo, U.M.; Zhao, B.T.; Kim, Y.H.; Kang, J.S.; Son, J.K.; Woo, M.H. Simultaneous analysis of seven marker compounds from Saposhnikoviae Radix, Glehniae Radix and Peucedani Japonici Radix by HPLC/PDA. Arch. Pharm. Res. 2016, 39, 695-704. [CrossRef] [PubMed]

18. Kim, H.M.; Jeong, S.Y.; Kim, S.M.; Lee, K.H.; Kim, J.H.; Seong, R.S. Simultaneous determination and recognition analysis of coumarins in Angelica decursiva and Peucedanum praeruptorum by HPLC-DAD. Nat. Prod. Sci. 2016, 22, 162-167. [CrossRef]

19. Tarmizi, A.A.A.; Wagiran, A.; Salleh, F.M.; Chua, L.S.; Abdullah, F.I.; Hasham, R.; Mostafiz, S.B. Integrated approach for species identification and quality analysis for Labisia pumila using DNA barcoding and HPLC. Plants 2021, 10, 717. [CrossRef]

20. Xu, H.; Li, P.; Ren, G.; Wang, Y.; Jiang, D.; Liu, C. Authentication of three source spices of Arnebiae Radix using DNA barcoding and HPLC. Front. Pharmacol. 2021, 12, 677014. [CrossRef] 
21. Zhong, Y.; Wang, H.; Wei, Q.; Cao, R.; Zhang, H.; He, Y.; Wang, L. Combining DNA barcoding and HPLC fingerprints to trace species of an important traditional Chinese medicine Fritillariae Bulbus. Molecules 2019, 24, 3269. [CrossRef]

22. Li, Y.P.; Geng, L.; Liu, Y.Y.; Chen, M.Y.; Mu, Q.R.; Zhang, X.; Zhang, Z.Y.; Ren, G.X.; Liu, C.S. Identification of three Daphne species by DNA barcoding and HPLC fingerprint analysis. PLOS ONE 2018, 13, 0201711. [CrossRef]

23. Zhang, Z.P.; Zhang, Y.; Zhang, Z.; Yao, H.; Liu, H.T.; Zhang, B.G.; Liao, Y.H. Comparative analysis of DNA barcoding and HPLC fingerprint to trace species of Phellodendri Cortex, an important traditional Chinese medicine from multiple sources. Biol. Pharm. Bull. 2016, 39, 1325-1330. [CrossRef]

24. Kim, J.H.; Doh, E.J.; Lee, G. Evaluation of medicinal categorization of Atractylodes japonica Koidz. by using internal transcribed spacer sequencing analysis and HPLC fingerprinting combined with statistical tools. Evid.-Based Complementary Altern. Med. 2016, 2016, 2926819.

25. Kim, J.H.; Doh, E.J.; Lee, G. Chemical differentiation of genetically identified Atractylodes japonica, A. macrocephala, and A. chinensis rhizomes using high-performance liquid chromatography with chemometric analysis. Evid.-Based Complementary Altern. Med. 2018, 2018, 4860371. [CrossRef]

26. Doh, E.J.; Lee, G.; Jung, H.J.; Kwon, K.B.; Kim, J.H. Chemotaxonomic monitoring of genetically authenticated Amomi Fructus using high-performance liquid chromatography-diode array detector with chemometric analysis. Molecules 2020, 25,4581 [CrossRef] [PubMed]

27. Vijayan, K.; Tsou, C.H. DNA barcoding in plants: Taxonomy in a new perspective. Curr. Sci. 2010, 99, $1530-1541$.

28. Zheng, L.; Watson, D.G.; Johnston, B.F.; Clark, R.L.; Edrada-Ebel, R.; Elseheri, W. A chemometric study of chromatograms of tea extracts by correlation optimization warping in conjunction with PCA, support vector machines and random forest data modeling. Anal. Chim. Acta 2009, 642, 257-265. [CrossRef]

29. Torniainen, P.; Popescu, C.-M.; Jones, D.; Scharf, A.; Sandberg, D. Correlation of studies between colour, structure and mechanical properties of commercially produced ThermoWood ${ }^{\circledR}$ treated Norway spruce and scots pine. Forests 2021, 12, 1165. [CrossRef]

30. Ratner, B. The correlation coefficient: Its values range between $+1 /-1$, or do they? J. Target. Meas. Anal. Mark. 2009, 17, 139-142. [CrossRef]

31. Schober, P.; Boer, C.; Schwarte, L.A. Correlation coefficients: Appropriate use and interpretation. Anesth. Analg. 2018, 126, 1763-1768. [CrossRef] [PubMed]

32. Batsukh, Z.; Toume, K.; Javzan, B.; Kazuma, K.; Cai, S.Q.; Hayashi, S.; Kawahara, N.; Maruyama, T.; Komatsu, K. Metabolomic profiling of Saposhnikoviae Radix from Mongolia by LC-IT-TOF-MS/MS and multivariate statistical analysis. J. Nat. Med. 2020, 74, 170-188. [CrossRef] [PubMed]

33. Fuchino, H.; Murase, S.; Hishida, A.; Kawahara, N. Simultaneous UHPLC/MS quantitative analysis and comparison of Saposhnikoviae radix constituents in cultivated, wild and commercial products. J. Nat. Med. 2021, 75, 499-519. [CrossRef]

34. Lajayer, B.A.; Ghorbanpour, M.; Nikabadi, S. Heavy metals in contaminated environment: Destiny of secondary metabolite biosynthesis, oxidative status and phytoextraction in medicinal plants. Ecotoxicol. Environ. Saf. 2017, 145, 377-390. [CrossRef]

35. Yang, L.; Wen, K.-S.; Ruan, X.; Zhao, Y.-X.; Wei, F.; Wang, Q. Response of plant secondary metabolites to environmental factors. Molecules 2018, 23, 762. [CrossRef]

36. Demasi, S.; Caser, M.; Lonati, M.; Cioni, P.L.; Pistelli, L.; Najar, B.; Scariot, V. Latitude and altitude influence secondary metabolite production in peripheral alpine populations of the mediterranean species Lavandula angustifolia Mill. Front. Plant Sci. 2018, 9, 983. [CrossRef]

37. White, T.J.; Bruns, T.; Lee, S.; Taylor, J.W. Amplification and direct sequencing of fungal ribosomal RNA genes for phylogenetics In PCR Protocols: A Guide to Methods and Applications; Innis, M.A., Gelfand, D.H., Sninsky, J.J., White, T.J., Eds.; Academic Press: New York, NY, USA, 1990; pp. 315-322.

38. Fay, M.F.; Swensen, S.M.; Chase, M.W. Taxonomic affinities of Medusagyne oppositifolia (Medusagynaceae). Keww Bull. 1997, 52, 111-120. [CrossRef]

39. Cuénoud, P.; Savolainen, V.; Chatrou, L.W.; Powell, M.; Grayer, R.J.; Chase, M.W. Molecular phylogenetics of caryophyllales based on nuclear $18 \mathrm{~S}$ rDNA and plastid rbcL, atpB, and matK DNA sequences. Am. J. Bot. 2002, 89, 132-144. [CrossRef] [PubMed]

40. Ford, C.S.; Ayres, K.L.; Toomey, N.; Haider, N.; Stahl, J.V.; Kelly, L.J.; Wikstrom, N.; Hollingsworth, P.M.; Duff, R.J.; Hoot, S.B.; et al. Selection of candidate coding DNA barcoding regions for use on land plants. Bot. Linn. Soc. 2009, 159, 1-11. [CrossRef]

41. Taberlet, P.; Gielly, L.; Pautou, G.; Bouvet, J. Universal primers for amplification of three non-coding regions of the chloroplast DNA. Plant Mol. Biol. 1991, 17, 1105-1109. [CrossRef]

42. Katoh, K.; Standley, D.M. MAFFT Multiple sequence alignment software version 7: Improvements in performance and usability. Mol. Biol. Evol. 2013, 30, 772-780. [CrossRef]

43. Criscuolo, A.; Gribaldo, S. BMGE (Block Mapping and Gathering with Entropy): A new software for selection of phylogenetic informative regions from multiple sequence alignments. BMC Evol. Biol. 2010, 10, 210. [CrossRef]

44. Guindon, S.; Dufayard, J.F.; Lefort, V.; Anisimova, M.; Hordijk, W.; Gascuel, O. New algorithms and methods to estimate maximum-likelihood phylogenies: Assessing the performance of PhyML 3.0. Syst. Biol. 2010, 59, 307-321. [CrossRef]

45. Lemoine, F.; Domelevo Entfellner, J.B.; Wilkinson, E.; Correia, D.; Dávila Felipe, M.; de Oliveira, T.; Gascuel, O. Renewing Felsenstein's phylogenetic bootstrap in the era of big data. Nature 2018, 556, 452-456. [CrossRef]

46. Lemoine, F.; Correia, D.; Lefort, V.; Doppelt-Azeroual, O.; Mareuil, F.; Cohen-Boulakia, S.; Gascuel, O. NGPhylogeny.fr: New generation phylogenetic services for non-specialists. Nucleic Acids Res. 2019, 47, W260-W265. [CrossRef] 
47. NCBI Resource Coordinators. Database resources of the National Center for Biotechnology Information. Nucleic Acids Res. 2016, 44, D7-D19. [CrossRef] [PubMed]

48. Han, B.X.; Yuan, Y.; Huang, L.Q.; Zhao, Q.; Tan, L.L.; Song, X.W.; He, X.M.; Xu, T.; Liu, F.; Wang, J. Specific PCR identification between Peucedanum praeruptorum and Angelica decursive and identification between them and adulterant using DNA barcode. Phcog. Mag. 2017, 13, 38-45. [PubMed] 